\title{
A psychological study of spinal cord injured patients involved in the Madras Paraplegia Project
}

O Somasundaram MBBS, FRC Psych, DPM, ${ }^{1} \mathrm{~S}$ Balakrishnan MA(Psychol), DM \& SP ${ }^{2}$ O S Ravindran MA (Psychol), ${ }^{3}$ T K Shanmugasundaram MS MChOrth FRCS(Eng), FRCS (Edin) FAMS ${ }^{4 *}$

${ }^{1}$ Professor of Psychiatry (retired), Madras Medical College; ${ }^{2}$ Clinical Psychologist, Madras Medical College and Government General Hospital, Madras; ${ }^{3}$ Research Scholar, University of Madras; ${ }^{4}$ Emeritus Professor of Orthopaedic Surgery, Madras Medical College, Madras, India.

The psychological features of spinal cord injured (SCI) patients involved in the Madras Paraplegia Project are described. Three hundred and twenty-eight patients were studied. Based on personality tests, $11 \%$ were extroverts, $14 \%$ were introverts and $76 \%$ were neither extroverts nor introverts. Twenty-four percent of the subjects were neurotic, $11 \%$ had a depressive illness, and $26 \%$ had pathological anxiety. The study has highlighted the psychological status of SCI patients, and the usefulness of a psychiatric team in the multidisciplinary care of such patients. This is probably the first large psychological study of SCI patients from a developing country.

Keywords: spinal cord injury; psychological aspects; Madras Paraplegia Project; personality inventory; depression; anxiety.

\section{Introduction}

Spinal cord injured (SCI) patients undergo severe physical, psychological and social stresses, and react in different ways to cope with stress. Whilst doing so they may exhibit psychological disturbances, some of which may interfere with their treatment and rehabilitation. Judd and Burrows ${ }^{1}$, and Guttmann $^{2}$ have described the psychological responses to severe spinal injury. The vital role of a psychiatric team in the multidisciplinary care of spinal cord injured patients is being increasingly recognised. ${ }^{1}$

In the Madras Paraplegia Project a qualitative improvement was observed in the assessment and care of patients requiring psychiatric and/or psychological assistance after the advent of a psychiatric team. The results of the psychological study of 328 patients admitted into the unit are described.

*Correspondence: 147, Periyar E.V.R. Road, Madras600010 , India.

\section{Materials and methods}

The Madras Paraplegia Project was started in 1978, and from February 1984 a psychiatrist and a psychologist joined the multidisciplinary team of the project.

The aims and the details of the management of patients involved in the Madras Paraplegia Project in the Government General Hospital, Madras has been reported by one of the authors (TKS). ${ }^{3-6}$ In Madras, the total care of SCI patients from the acute stage through to the rehabilitation stage was carried out by a multidisciplinary team in a general hospital. From June 1979 to December 1986, 939 patients were treated. An analysis of 56 variables concerning these patients has previously been published. ${ }^{4}$

The present study consists of $328 \mathrm{SCI}$ patients who were admitted to the Madras Paraplegia Project from February 1984 to December 1986. Patients with the following criteria were excluded from the study: (a) those with a high cervical spine injury, those with multiple injuries, and patients admitted in a moribund condition or who died soon after admission; (b) patients who had a 
spinal operation soon after admission; (c) patients who did not speak the Tamil language.

After obtaining a detailed history, all of the patients had a psychological/psychiatric assessment within 10 to 30 days of admission, most within the first 2 weeks of the injury. The following psychological tests were administered to all the patients:-

1 Eysenck Personality Inventory (EPI). ${ }^{7}$ EPI consists of 57 items of which 24 items are for extroversion dimensions of the personality, 24 items for neuroticism and the remaining 9 items are a measure of lie scale. Scores of 17 and above are classified as high extroversion, 8 and below are indicative of introversion and scores of 9 to 16 are grouped as neither introversion nor extroversion.

2 Beck's Depression Inventory (BDI). ${ }^{8}$ BDI consists of 24 items with a maximum score of 72 . Scores below 20 are categorised as low, 21 to 30 as mild, 31 to 40 as moderate and 41 and above as severely depressed.

3 Taylor's Manifest Anxiety Scale (TMAS). ${ }^{9}$ TMAS consists of 50 items for measuring anxiety with a maximum score of 50 . The score of 0 to 20 is classified as low, 21 to 30 as high and those who score 31 and above come under the category of high and uncontrollable anxiety.

To ensure reliable communication with the patients, Tamil versions of these inventories have been used in the current study. Several authors have reported the usefulness of the standardised Tamil version of these tests in South Indian patients. ${ }^{10-14}$ The categories arrived at from the scores are used in the discussion. The members of the multidisciplinary team other than the psychiatric team were thus able to understand the commonly used terms of the categories better than the scales or the scores of the inventories.

\section{Description of the series}

Over $50 \%$ of the patients were under the age of 30 years $(\mp 32.26 \mathrm{SD} \pm 12.86)$. The study group consisted of 296 male and 32 females. Sixty-four percent of the patients were married and $23 \%$ were single. There was one marital separation; and the marital status of $11 \%$ was not available. Fifty-five percent of the patients belonged to the lower socioeconomic group with incomes less than 300 rupees per month. Thirty-eight percent of the series were illiterate, $38 \%$ had had some education, and $2.3 \%$ were graduates. For the illiterate patients the inventories were read out and the answers were recorded. Information regarding educational status was not available for $19 \%$ of the patients. Sixty percent of the patients were unskilled agricultural labourers, and only $10 \%$ were skilled workers.

\section{Preinjury psychiatric disorders}

Three patients had evidence of preinjury psychiatric disorders at the initial interview. A male patient had schizophrenia and had jumped from a height in a suicidal attempt. Another male patient jumped from a height during a long session smoking marijuana. The long-suffering spouse of an alcoholic jumped from her flat. Many of the women who had 'accidentally' fallen into wells had disturbed family relations. These patients were adequately treated for their respective psychiatric illness.

\section{Discussion and results}

\section{Personality traits (Table I)}

Eleven percent of the study population were extroverts, $14 \%$ were introverts, $76 \%$ were neither introverts nor extroverts, and $24 \%$ had neuroticism. The scores on the Eysenck Personality Inventory failed to define any specific psychological characteristic in SCI patients. However, the preinjury personality and the means of coping with stress became prominent after the spinal injury. Patients with high neuroticism benefit from supportive psychotherapy. Grundy and Russell ${ }^{15}$ have explained the difficulties likely to be encountered in the rehabilitation of these patients.

The frustration associated with severe physical limitations are accentuated by the fact that most of the patients were young 
Table I Eysenck Personality Inventory

\begin{tabular}{lcccc}
\hline & Introverts (\%) & $\begin{array}{c}\text { Extraversion } \\
\text { Neither introverts nor } \\
\text { extroverts (\%) }\end{array}$ & Extroverts (\%) & Total \\
\hline $\begin{array}{l}\text { No of patients } \\
\text { Scores }\end{array}$ & $\begin{array}{c}45(13.7) \\
1-8\end{array}$ & $\begin{array}{c}248(75.6) \\
9-16\end{array}$ & $\begin{array}{c}35(10.6) \\
17-24\end{array}$ & 328 \\
\hline & Low (\%) & Medium (\%) & High (\%) & \\
\hline No of patients & $66(20.1)$ & $183(55.7)$ & $79(24)$ & 328 \\
\hline
\end{tabular}

and had led an active life before the injury. The sudden disablement and the need to lead a more dependent life can lead to the necessity for prolonged adjustment. Steward ${ }^{16}$ has likened the delay to that of the development of a sense of identity in adolescence noted by Erickson ${ }^{17}$ This observation has been recorded by Cogswell ${ }^{18}$ and Felton \& Littman. ${ }^{19}$

\section{Depression and anxiety (Tables II and III)}

Approximately $70 \%$ of the patients had minimal or only mild depression on the Beck Depression Inventory, whereas only $11 \%$ showed marked depression. Twentysix percent had severe anxiety on the Taylor Manifest Anxiety Scale. The incidence of depressive illness in our patients at Madras was similar to the observations of Judd \& Brown $^{20}$ in Australia. Few systematic studies have confirmed that significant depress- ive illness was present only in a minority of SCI patients. ${ }^{21-23}$

In addition to the physical and psychological problems, our patients had also to contend with several socioeconomic problems. As indicated above, many were illiterate unskilled labourers who were the main breadwinners in the family. Social security and insurance in any form were not available.

The patients with depression and anxiety were treated with psychopharmacological agents (tricyclic antidepressants and anxiolytic agents, mainly amitryptiline and diazepam). Patients with neuroticism had supportive psychotherapy. Many of the psychological stresses were identified and appropriate therapeutic measures were taken. The patients with psychological disturbance had prolonged observation and follow ups lasting for 2 to 3 years. However, financial and socioeconomic constraints influenced the effective follow up of all of the patients.

Table II Beck Depression Rating Scale

\begin{tabular}{lccccc}
\hline & Low (\%) & Mild (\%) & Moderate (\%) & Severe (\%) & Total \\
\hline No of patients & $130(39.6)$ & $96(29.2)$ & $65(19.8)$ & $37(11.2)$ & 328 \\
Scores & $1-20$ & $21-30$ & $31-40$ & $41-72$ & \\
\hline
\end{tabular}

Table III Taylor's Manifest Anxiety Scale

\begin{tabular}{lcccc}
\hline & Low (\%) & High \& tense (\%) & High \& uncontrolled (\%) & Total \\
\hline No of patients & $133(40.5)$ & $109(33.2)$ & $86(26.2)$ & 328 \\
Scores & $0-20$ & $21-30$ & $31-50$ & \\
\hline
\end{tabular}


Other members of the multidisciplinary team found significant inprovement in the patients' compliance for their respective modalities of management.
To the best of our knowledge, the present study would appear to be the first large psychological study of SCI patients from a developing country.

\section{References}

1 Judd FK, Burrows GD (1986) Liaison psychiatry in a spinal injuries unit. Paraplegia 24: 6-19.

2 Guttmann L (1976) Spinal Cord Injuries - Comprehensive Management and Research. Blackwell Scientific Publications, Oxford: 506.

3 Shanmugasundaram TK (1983) The philosophy of care and the Madras Model. Proceedings of Indo-USA dialogue on total care of spinal cord injured patients, Madras: 8-11.

4 Shanmugasundaram TK (1984) Final report of PL 480 Paraplegia Project, Madras.

5 Shanmugasundaram TK (1987) The Challenge and the Response. A Primer of SCI Care in a General Hospital PL480 Paraplegia Project, Madras.

6 Shanmugasundaram TK (1988) The care of SCI patients in the developing nations - can we stem the rot? Paraplegia 26: $10-11$.

7 Eysenck HJ, Eysenck SBG (1964) Manual of the Eysenck Personality Inventory. Univerisity of London Press, London.

8 Beck AT, Ward CH, Mendelson M, Mock J, Erbaugh J (1961) An inventory for measuring depression. Arch Gen Psychiatry 4: 561-571.

9 Taylor JA (1953) A personality scale of manifest anxiety. J Abnormal Psychol 48: 285-290.

10 Abraham A, Sundar Rao PS, Verghese A (1977) Standardization of vernacular translations of the Eysenck Personality Inventory. Indian J Psychiatry 19(2): 60-66.

11 Shunmugham TE (1979) Extraversion-Intraversion and neuroticism factors in bronchial asthma, peptic ulcer and ischaemic heart disease. Indian J Psychiatry 21: 138-141.

12 Kuruvilla K (1983) Translation of Beck Depression Inventory into 3 South Indian languages. Indian J Psychol Med 4: 77-81.

13 Rangaswami K (1980) Treatment of anxiety state in elderly persons by behaviour therapy. Indian J Psychol Med 3: 139-142.

14 Rangaswami K (1981) Treatment of chronic nervous diarrhoea by desensitisation - a case report. Indian $J$ Psychol Med 4: 77-81.

15 Grundy D, Russell J (1986) ABC of spinal cord injury - later management and complications. $\mathrm{Br} \mathrm{Med} J$ 292: 743 .

16 Stewart TD (1977) Coping behaviour and the moratorium following spinal cord injury. Paraplegia 15: 338-342.

17 Erickson EH (1965) Childhood and Society. Norton, New York: 222-251.

18 Cogswell BE (1968) Self-socialisation - the adjustment of paraplegia in the community. J Rehabil 34: 11-13.

19 Felton JS, Litman M (1965) Study of employment of 222 men with spinal cord injury. Arch Phys Med Rehabil 46: 809-814.

20 Judd FK, Brown DJ (1987) Psychiatry in the spinal cord injuries unit. Paraplegia 25: 254-257.

21 Lawson N (1978) Significant events in the rehabilitation process: the spinal cord injured patients' point of view. Arch Phys Med Rehabil 59: 573-579.

22 Fullerton DT, Harvey RF, Klein MH, Howell T (1981) Psychiatric disorders in patients with spinal cord injury. Arch Gen Psychiatry 38: 1369-71.

23 Judd FK, Burrows GD, Brown DJ (1986) Depression following acute spinal cord injury. Paraplegia 24: $358-364$. 